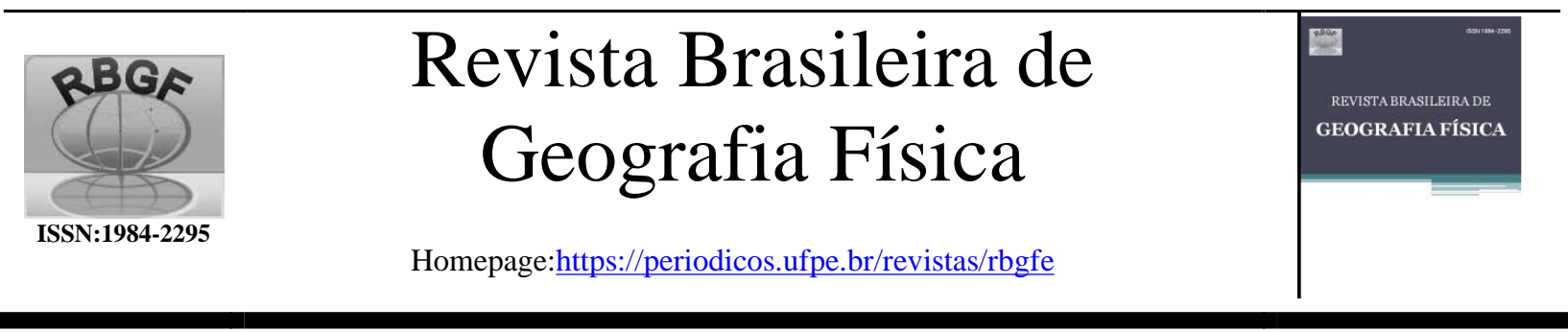

\title{
Avaliação da evapotranspiração estimada pelo MODIS e do balanço hídrico para a bacia do Pontal-Pernambuco
}

\author{
${ }^{1}$ Pedro Vinicius de Brito, ${ }^{2}$ Rodrigo de Queiroga Miranda, ${ }^{3}$ Ygor Cristiano Brito Morais, \\ ${ }^{4}$ Valéria Sandra de Oliveira Costa, ${ }^{5}$ Magna Soelma Beserra de Moura, ${ }^{6}$ Antonio Samuel Alves da Silva, \\ ${ }^{7}$ Josiclêda Domiciano Galvincio \\ ${ }^{1}$ Bolsista de PIBIC do CNPq. Universidade Federal Rural de Pernambuco.E-mail: pedro brito1997@hotmail.com. \\ ${ }^{4}$ Professora colaboradora do Programa de Pós-Graduaçao em Desenvolvimento e Meio Ambiente da Universidade \\ Federal de Pernambuco -PRODEMA/UFPE. E-mail: costavso@yahoo.com.br. ${ }^{7}$ Professora do Departamento de \\ Ciências Geográficas da Universidade Federal de Pernambuco e do PRODEMA/UFPE. Autor Correspondente \\ (josicleda.galvincio@ufpe.br)
}

\begin{abstract}
R E S U M O
A região semiárida do nordeste do Brasil tem forte tendência de diminuição da precipitação com as mudanças climáticas. Sabe-se que os impactos sociais, econômicos e ambientais serão muito altos se isso vier acontecer. A evapotranspiração e o balanço hídricos são variáveis importantes na tomada de decisão de políticas públicas relacionadas a chuva e recursos hídricos, uma vez que possibilita dar maior conhecimento do fornecimento de á gua em uma determinada região. Assim, avaliar diferentes metodologias de estimativas dessas variáveis proporciona uma importante contribuição para o planejamento de políticas públicas ambientais. Diante do exposto, este estudo tem o objetivo de a valiar a evapotranspiração estimada com MODIS e balanço hídrico obtido com o Soil Wat er Assessement Tools (SWAT) para a bacia do Pontal, Pernambuco, Brasil, para o ano de 2016. Os resultados mostram a grande dependência da evapotranspiração reale do escoamento superficial ao volume de chuva da bacia. Foi possível constatar, que as áreas de caatinga preserva das a presentaram as maiores evapotranspiração ao longo do a no. Destaca -se ta mbém o volume de água armazenado no solo nessas áreas. O maior volume escoado esteve presente na agropecuária e solo exposto. Esses resultados mostram os impactos das ações antrópicas sobre os recursos natura is da bacia do Pontal que podem acarretar maiores complicações na cobertura solo e balanço hídrico da região.
\end{abstract}

Palavras-chave: Modelagem, sensoriamento remoto, SWAT, MODIS.

\section{Evaluation of evapotranspiration estimated by MODIS and water balancefor the Pontal-Pernambuco basin}

\section{A B S T R A C T}

The semi-arid region of northeastern Brazil has a strong tendency to decrease precipitation with climate change. It is known that the social, economic, and environmental impacts will be very high if this happens. Evapotranspiration and water balance are important variables in public policy decision-making related to rain and water resources, as it allows for greater knowledge of the water supply in each region. Thus, evaluating different estimation methodologies for these variables provides an important contribution to the planning of environmental public policies. Given the above, this study aims to evaluate the evapotranspiration estimated with MODIS and the water balance obtained with the Soil Water Assessment Tools (SWAT) for the Pontal basin, Pernambuco, Brazil, for the year 2016. The results show the great dependence of actualevapotranspiration and runoff on the volume of rainfall in the basin. It was possible to verif $y$ that the preserved caatinga a rea s had the highest evapotranspiration throughout the year. Also noteworthy is the volu me of water stored in the soil in these areas. The largest volume drained was present in agriculture and livestock and exposed soil. These results show the impacts of anthropic actions on the natural resources of the Pontalbasin, which can lead to greater complications in the region's land cover and water balance.

Keywords: Modeling, remote sensing, SWAT, MODIS.

\section{Introdução}

Com grande diversidade agroecológica e diferentes formas de vida, o planeta Terra é ocupado por mais de sete bilhões de pessoas (Tyagi, 2020). As suas regiões enfrentam dificuldades de escassez de água, agravadas pelo

Brito., P., V., Miranda., R., Q., Morais., Y., C., B., Costa., V., S., O., Moura., M., S., B., Silva., A., S., A., Galvincio., J., D. 
Revista Brasileira de Geografia Física v.14, n.05 (2021) 2866-2877.

aumento populacional e pelas mudanças climáticas (Wang, 2021). Por conta dos impactos globais da escassez hídrica nos meios de vida humanos e nos sistemas naturais, diversos programas globais, como exemplo, as Nações Unidas, buscam melhorar o acesso humano à água, tentado obter um ecossistema com maio sustentabilidade (Bond, 2019).

Desta forma, inúmeros estudos são desenvolvidos com o propósito de esclarecer diferentes mecanismos que amenizem os impactos climáticos de uso e ocupação do solo, Silva et al. (2013), Morais et al. (2017), Miranda et al. (2018) e Lima et al. (2021), além disso, o aumento populacional e das mudanças climáticas nos recursos hídricos Galvincio (2021), Galvincio et al., (2021). No entanto, para a realização desses estudos, pesquisadores encontram dificuldades diversas, tais como a coleta de dados e, grandes mudanças na ocupação e utilização do solo (Galvincio, 2019; Galvincio e Naue, 2019).

Com o provável aumento de secas severas ocasionadas por mudanças climáticas no Brasil e em diversos lugares no mundo, ampliou-se a necessidade de antecipar ações para superar possíveis impactos (Gutiérrez et al., 2014). Em destaque a escasseeis de água no Brasil, te mos a região semiárida do Nordeste, que nas últimas décadas passou por uma das secas mais severas e extensas já registrada região (Santos e Latrubesse, 2021).

A região Nordeste abrange uma área de $1.600 .000 \mathrm{~km}^{2}$ do território nacional, sendo que $86 \%$ representa zona semiárida, onde a vegetação dominante é a caatinga, que ocupa uma área de $844.453 \mathrm{Km}^{2}$, representando cerca de $70 \%$ da região Nordeste e $11 \%$ do todo território nacion al (Marengo, 2010; MMA, 2012).

Com o objetivo de instaurar planos de manejo apropriados que garantam a diversidade biológica e serviços ecossistêmicos proporcionados pela caatinga, políticas socioambientais foram criadas pelo governo do estado de Pernambuco. Em destaque, temos a Lei $\mathrm{N}^{\circ} 14.090$, de 17 de junho de 2010, que Institui a Política Estadual de Enfrentamento às Mu dan ça s Climáticas, e a Lei no 14.091 , de 17 de junho de 2010, que Institui a Política Estadual de Combate à Desertificação e Mitigação dos Efeitos da Seca.

Embora a caatinga seja a maior e mais rica em biodiversidade das florestas tropicais sazonalmente seca do planeta (Silva, Leal e Tabarelli, 2018), e existente apenas no Brasil, é ainda considerada uma área com poucos estudos realizados (Vieira et al. 2016). Com o objetivo de solucionar este problema, pesquisadores do mundo todo têm desenvolvido diversos modelos matemáticos que relacionam solo, planta e atmosfera, como o modelo Surface Energy Balance for Land (SEBAL) por Bastiaanssen (1995), de Gash por Gash (1979) e Soil and Water Assessment Tool (SWAT) por Arnold et al. (1998).

Segundo Arnold et al. (1998), modelos matemáticos são aplicados por funcionários do governo e pesquisadores do mundo todo com o objetivo de simular processos biológicos e físicos, que por conta do alto custo associado ou complexidade, acima de tudo em monitoramento s constantes em grande escala, passam por dificuldades analíticas. De acordo com Uzeika et al. (2012), os modelos matemáticos têm mostrado uma imensa potencialidade como instrumento de suporte ao planejamento de uso da terra, tendo a finalidade de gerar melhorias na qualidade do solo e da água.

O SWAT é um dos modelos matemátic os e hidrológicos mais utilizados na atualidade, e tem a finalidade de simular impactos possíveis de uso e ocupação do solo nos recursos hídricos e a produção de sedimentos e aplicação de produtos químicos nas plantações no interior dos grandes complexos de bacias hidrográficas, inúmeros tipos de solo, condições de gestão, e utilização do terreno no decorrer de um longo intervalo de tempo (Arnold et al., 1998).

Por outro lado, observa-se um aumento na necessidade de aplicar modelos hidrológicos em bacias hidrográficas, nas quais não se tem uma rede de monitoramento fluviométrico ou a rede existente dispõe de pouco tempo de instalação (Saraiva, Fernandes e Naghettini, 2011). De acordo com (Melesse et al., 2007), com a disponibilidade de dados de sensoriamento remoto de inúmeros sensores de diversas plataformas com extenso conjunto de resoluções espaciotemporais, espectrais e radiométricas, o sensoriamento remoto talvez tenha se tornado a melhor fon te de dados para estudos e aplicações em grande escala.

Segundo Novo (2010), o sensoriamento remoto é a junção de ferramentas que proporciona obter características de processos físicos, químicos e biológicos através da análise de suas interações com a radiação eletromagnética (REM) utilizando sensores. Dentre os diversos sens ores apresentados na literatura, o sensor MODIS possibilita visualizar toda a superfície da terra de um a dois dias, sendo apropriado na recuperação de variáveis de vegetação e água em larga e scala (Teixeira et al., 2012). Os autores ainda afirmam

Brito., P., V., Miranda., R., Q., Morais., Y., C., B., Costa., V., S., O., Moura., M., S., B., Silva., A., S., A., Galvincio., J., D. 
Revista Brasileira de Geografia Física v.14, n.05 (2021) 2866-2877.

que os modelos aplicados com imagens de satélite do MODIS são adequados para avaliar a produtividade hídrica e estimar os impactos da ampliação de áreas irrigadas sobre a vegetação nativa no uso regional de água em casos de mudanças aceleradas no padrão de utilização do solo.

Diante do exposto, o objetivo deste estudo é utilizar o modelo SWAT calibrado para a bacia do Pontal e avaliar a variação espacial e temporal da evapotranspiração e do escoamento superficial da bacia do Pontal para o ano de 2016.

\section{Material e métodos}

\section{Área de estudo}

O presente estudo foi realizado na unidade de planejamento hídrico UP13, que corresponde à bacia do riacho do Pontal (Figura 1), composta por 59 sub-bacias, localizada no extremo oeste do estado de Pernambuco, região semiárida pernambucana do nordeste brasileiro, inserida na mesorregião do São Francisco, com distância de $734 \mathrm{~km}$ de Recife a capital (APAC, 2021).

$A$ área de drenagem do rio abrange quatro municípios, estando o município de Afrânio totalmente inserido nabacia, os de Dormentes e Lagoa Grande com suas sedes inseridas na bacia, e o de Petrolina parcialmente inserida da bacia. Totalizando $6.015,33 \mathrm{~km}^{2}$, o equivalente a $6,12 \%$ da área absoluta do estado, com as coordenadas geográficas entre $08^{\circ} 19^{\prime} 00^{\prime \prime}$ e $09^{\circ} 13^{\prime} 24^{\prime \prime}$ Sul e $40^{\circ} 11^{\prime} 42^{\prime \prime}$ e $41^{\circ} 20^{\prime} 39^{\prime \prime}$ Oeste (APAC, 2021).

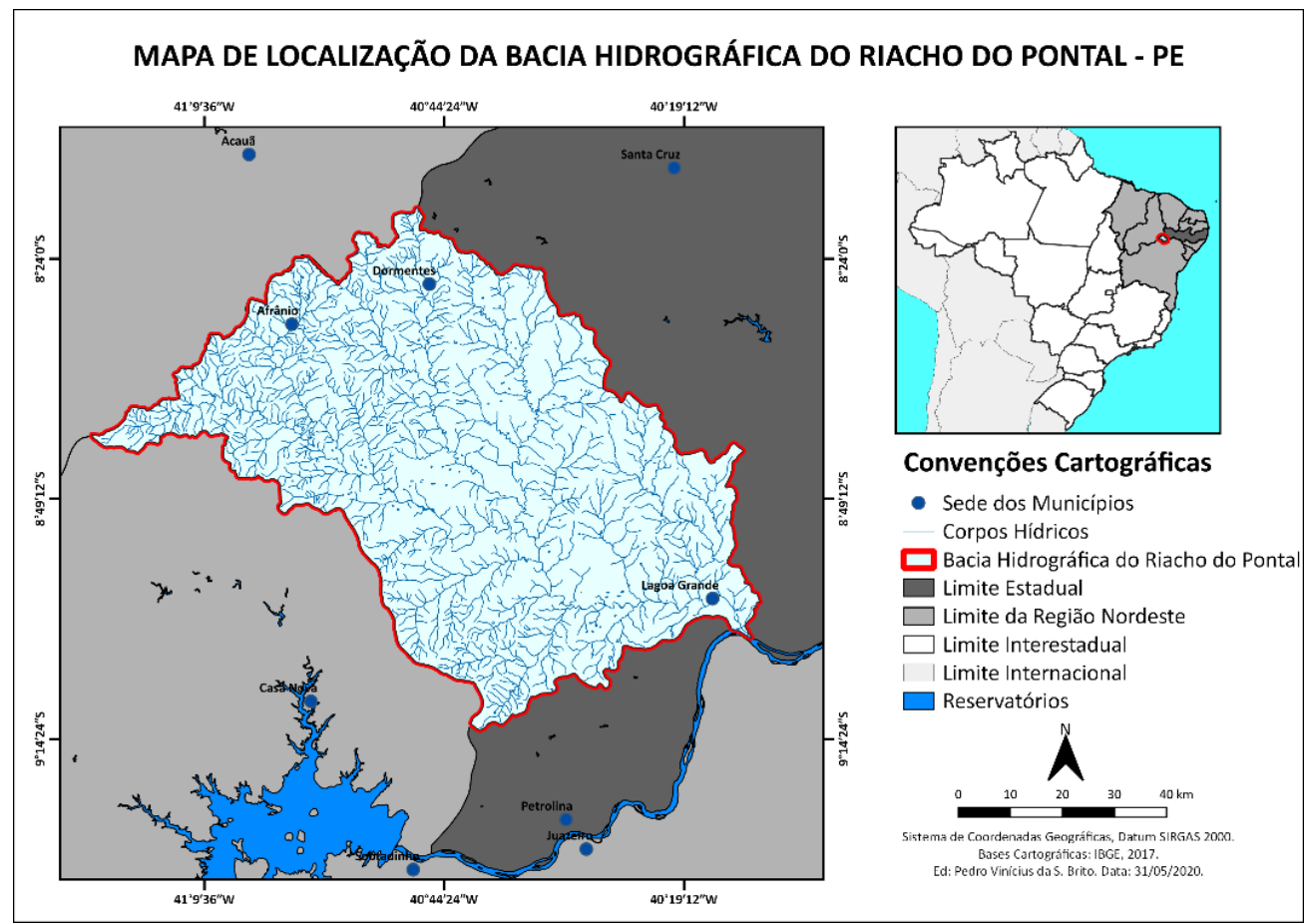

Figura 1. Localização geográfica do município de Petrolina, Pernambuco.

É possível observar na região de estudo (Figura 2), que de acordo com a distribuição das classes de uso do solo, a vegetação de caatinga e o solo exposto e/ou área urbana predominam a região. Próximo ao rio São Francisco, ficam os cultivos irrigados, que representam uma pequena parte da área total da bacia.

Brito., P., V., Miranda., R., Q., Morais., Y., C., B., Costa., V., S., O., Moura., M., S., B., Silva., A., S., A., Galvincio., J., D. 
Revista Brasileira de Geografia Física v.14,n.05 (2021) 2866-2877.

MAPA DE USO E COBERTURA DO SOLO DA BACIA HIDROGRÁFICA DO RIACHO DO PONTAL - PE
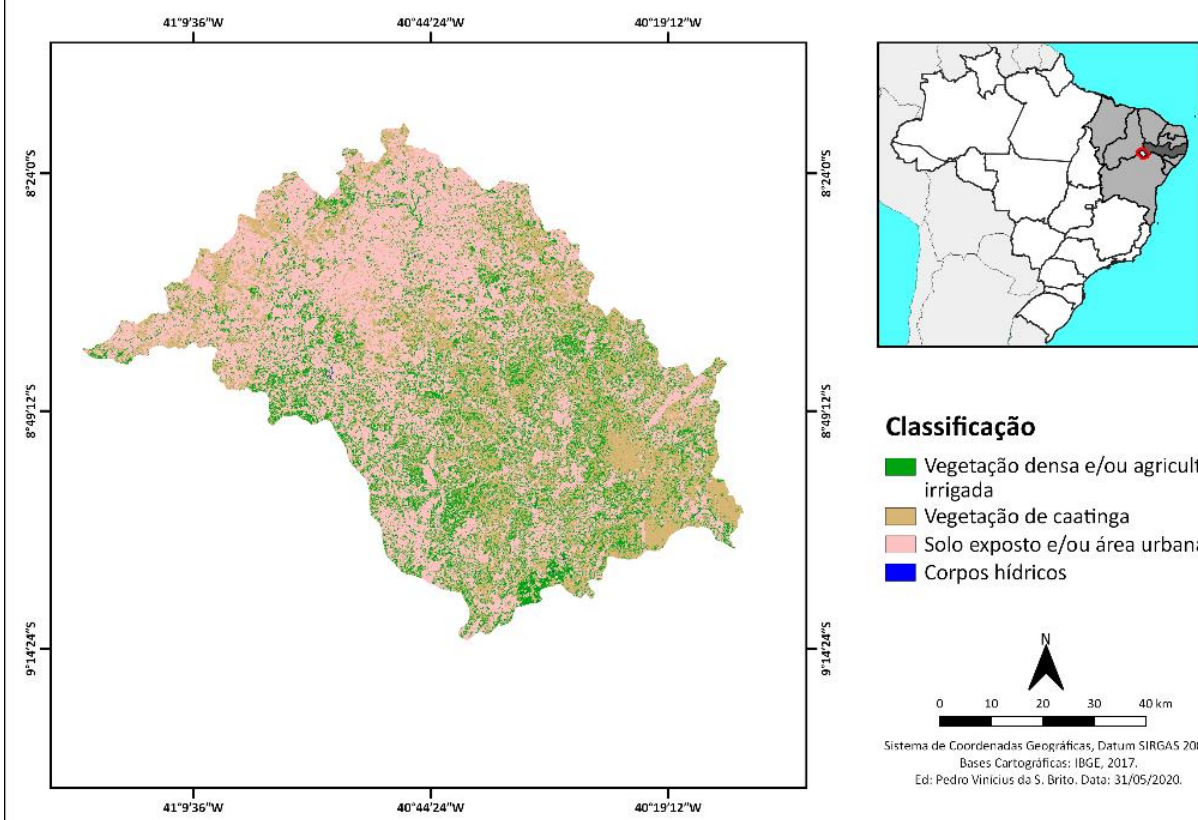

Classificação

Vegetação densa e/ou agricultura irrigada

$\square$ Vegetação de caating

$\checkmark$ Solo exposto e/ou área urbana

Corpos hídricos

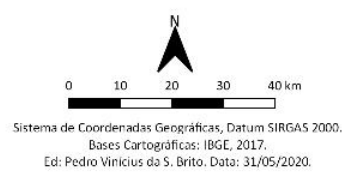

Figura 2. Mapa de uso e ocupação do solo do município de Petrolina, Pernambuco.

O modelo hidrológico Soil Water Assessment Tools (SWAT) desenvolvido nos domínios da Texas A\&M University (Arnold et al. 1998), foi utilizado para simular o impacto das alterações ocorridas na utilização do solo na bacia do riacho do Pontal, Pernambuco, ao longo do ano de 2016. Esse modelo está inserido no Sistema de Unidade de Respostas Hidrológicas para Pernambuco (SUPer), que é disponibilizado de maneira gratuita pelo site https://super.hawqs.tamu.edu/\#/, e é uma ferramenta de avaliação de bacias hidrográfic as para o estado de Pernambuco, sendo constituído de um avançado sistema de modelagem da quantidade e qualidade de água.

O SUPer dispõe de um banco de dados, interface, e modelos hidrológicos que estão sendo desenvolvidos e calibrados para as bacias hidrográficas do estado para avaliar os impactos do manejo do solo, da poluição hídrica e das mudanças climáticas sobre a quantidade e qualidade da água dos rios e reservatórios de Pernambuco.

Foram analisados os dados da evapotranspiração do sensor MODIS produto MOD16A2 para o ano de 2016. Em seguida, estes dados foram comparados com os dados simulados do modelo SWAT que já se encontra calibrado para a bacia do Pontal por Miranda (2017), e no mesmo modelo, também foi avaliado o escoamento superficial da bacia.

\section{Modelo SWAT}

$\mathrm{O}$ modelo SWAT é fundamentado em uma estrutura de controles para reproduzir o escoamento, sedimentos e agroquímicos pelo interior da bacia (Fernandes, Galvíncio e Freire, 2012). De acordo com Arnold et al. (2012), existe no SWAT cinco componentes principais, que são, clima, vegetação, manejo da terra, solo e hidrologia. No componente hidrológico do modelo SWAT, tem-se a percolação, as subrotinas do escoamento superficial, o e scoamento lateral subsuperficial, o fluxo de retorno do aquífero raso e a evapotranspiração (Fernandes, Galvíncio e Freire, 2012).

O modelo SWAT tem como base do ciclo hidrológico a equação do balanço hídrico, apresentada na Equação 1.

$$
\begin{aligned}
S W_{f}= & S W_{i}+\sum_{t=1}^{t}\left(P-Q_{s}-E T-\right. \\
& \left.W_{S}-Q_{g w}\right)
\end{aligned}
$$

Onde: $S W_{f}$ e $S W_{i}$ é a quantidade de água no solo inicial e final (mm), $t$ é tempo (dias), $P$ é precipitação $(\mathrm{mm}), Q_{S}$ é escoamento superficial $(\mathrm{mm}), E T$ é evapotranspiração $(\mathrm{mm}), W_{s}$ é percolação (mm) e $Q_{g w}$ é fluxo de base ( $\mathrm{mm}$ ).

$\mathrm{O}$ escoamento superficial vem sendo utilizado regulamente desde o ano de 1950 , ele é simulado com um modelo prático conhecido como Soil Conservation Service (ou curva SCS). Segundo Rallison e Miller (1981), este modelo 
Revista Brasileira de Geografia Física v.14, n.05 (2021) 2866-2877.

tem a finalidade de servi como base única para estimar em diversas coberturas da terra e classe de solo o escoamento superficial.

A evapotranspiração é o segundo componente principal no estudo de verificação do ciclo da água, tal que, compara as perdas de hídricas para a atmosfera por meio da transpiração da vegetação e evaporação do solo (Brutsaert, 2005; Ferreira et al., 2021).

No modelo SWAT, a evapotranspiração real (ETr) é estimada após a obtenção dos valores de evapotranspiração potencial total (ETp), que é a simulação em que a evapotranspiração aconteceria em uma dada área extensa totalmente e igualmente revestida de vegetação em processo de desenvolvimento, comfornecimento de água não cerceado na superfície. O SWAT inicia evaporando toda a água acumulada pelos dosséis das plantas, posteriormente, estima a maior quantidade de transpiração e sublimação/evapotranspiração do solo utilizan do cálculo similar ao de Ritchie (1972), e assim, a sublimação real e evapotranspiração real da superfície são estimadas (NEITSCH et al., 2011b).

\section{Processamento das imagens}

Foram utilizados produtos de evapotranspiração (ET) do sensor MODIS abordo do satélite Terra (MOD16A2), com resolução espacial de $1 \mathrm{~km}$ e resolução temporal de oito dias para o ano de 2016, totalizando 94 imagens, pois foi necessário utilizar duas imagens do mesmo dia para cobrir toda a região da bacia. Esses produto s estão disponíveis de maneira gratuita no site do serviço Geológico dos Estados Unidos USGS/NASA. Na Tabela 1, apresenta-se a descrição do produto MOD16A2.

Tabela 1. Descrição das bandas do produto MOD16A2.

\begin{tabular}{cccc}
\hline Banda & Descrição & Unidades & Fator de escala \\
\hline 1 & Evapotranspiração & $\mathrm{mm} / 8$ dias & 0,1 \\
2 & Fluxo de calor latente & $\mathrm{J} / \mathrm{m}^{2}$ dias & $10^{4}$ \\
3 & Evapotranspiração potencial & $\mathrm{mm} / 8$ dias & 0,1 \\
4 & Fluxo de calor latente potencial & $\mathrm{J} / \mathrm{m}^{2}$ dias & $10^{4}$ \\
\hline
\end{tabular}

As imagens baixadas, inicialmente vem no formato HDF, para utilizá-las foi necessário convertê-las ao formato TIF. Para isto, u sou-se a ferramenta Modis Reprojection Tool (MRT) que também já fez o mosaico das imagens, e que se encontra disponível para Download gratuitamente na página do serviço Geológico dos Estados Unidos USGS/NASA.

Em seguida, foi efetuada a conversão do s valores parcialmente processados para as estimativas reais na superfície, multiplicando esses valores parciais pela correção 0,1 . Depois, foram retirados das imagens, os pixels que continham nuvens, considerados ruins, fez-se o acumulado mensal e o recorte da bacia. Com isso, confeccionou-se mapas temáticos de evapotranspiração mensal, totalizando 12 imagen s (janeiro-dezembro). Ambos os passos, foram realizados através do software de geoprocessamento. Posteriormente, foram extraídos os valores de evapotranspiração mínima, média e o desvio padrão para os dados mensais de 2016. Deste modo, foi avaliada a variação espacial e temporal da evapotranspiração.
Segundo Mu et al. (2007) o produto de evapotranspiração é formado pelos elementos de sensores remotos e dados meteorológicos, calculados partir da utilização do algoritmo apresentado pela Equação 2, que é reescrito com base na equação mostrada por Cleugh et al. (2007).

$$
E T=\frac{\Delta\left(R_{n}-G\right)+\frac{\rho C_{p}\left(e_{s}-e_{a}\right.}{r_{a}}}{\Delta+\gamma\left(1+\frac{r_{s}}{r_{a}}\right)}
$$

Na qual a variável $R_{n}$ é o saldo de radiação, $G$ é o fluxo de calor do solo, $\rho$ é a densidade do ar, $C_{p}$ é o calor específico do ar a uma pressão constante, $(1013106 \mathrm{~J} \mathrm{~kg} \mathrm{-} 1 \mathrm{~K}-$ $1), e_{s}$ é a pressão de saturação do vapor, $e_{a}$ é a pressão de vapor no momento, $\Delta$ é a inclinação curva de pressão em relação à temperatura do ar, e $\gamma$ é a constante psicométrica.

Brito., P., V., Miranda., R., Q., Morais., Y., C., B., Costa., V., S., O., Moura., M., S., B., Silva., A., S., A., Galvincio., J., D. 
Simulação do escoamento superficial e evapotranspiração com modelo SWAT

A simulação do escoamento superficial e evapotranspiração (ET) para o ano de 2016, foi realizada no SWAT, software inserido no SUPer, já calibrado para a bacia estudada, que contém um banco de dados climáticos inserido e disponível para a simulação de 2010 a 2016, a fim de obter estimativas do escoamento superficial e avaliar a variação temporal e espacial do escoamento e disponibilidade hídrica.

\section{Resultados e discussão}

Valores médios mensais de Precipitação obtidos a partir do Modelo SWAT

$\mathrm{Na}$ Figura 3 está apresentada à sazonalidade da precipitação pluviométrica mensal estimada pelo SWAT para a bacia do pontal ao longo do ano 2016. Pode-se observar que foi irregular, concentrada no mês de janeiro, e entre os meses de janeiro a março ocorreram os maiores volumes de chuva, embora em julho e agosto houveram baixos volumes, seguido de um longo período de estiagem entre meados de maio a novembro, situação comum na região semiárida.

De acordo com Silva (2016), o alto estresse hídrico observado na Bacia Hidrográfic a do Riacho do Pontal mostra a impossibilidade da da mesma fornecer água sufuciente $\mathrm{p}$ ara atender as necessidades da região, de modo que a sua integração com a Bacia Hidrográfica do Rio São Francisco é a possibilidade mais viável para a realidade apresentada na área.

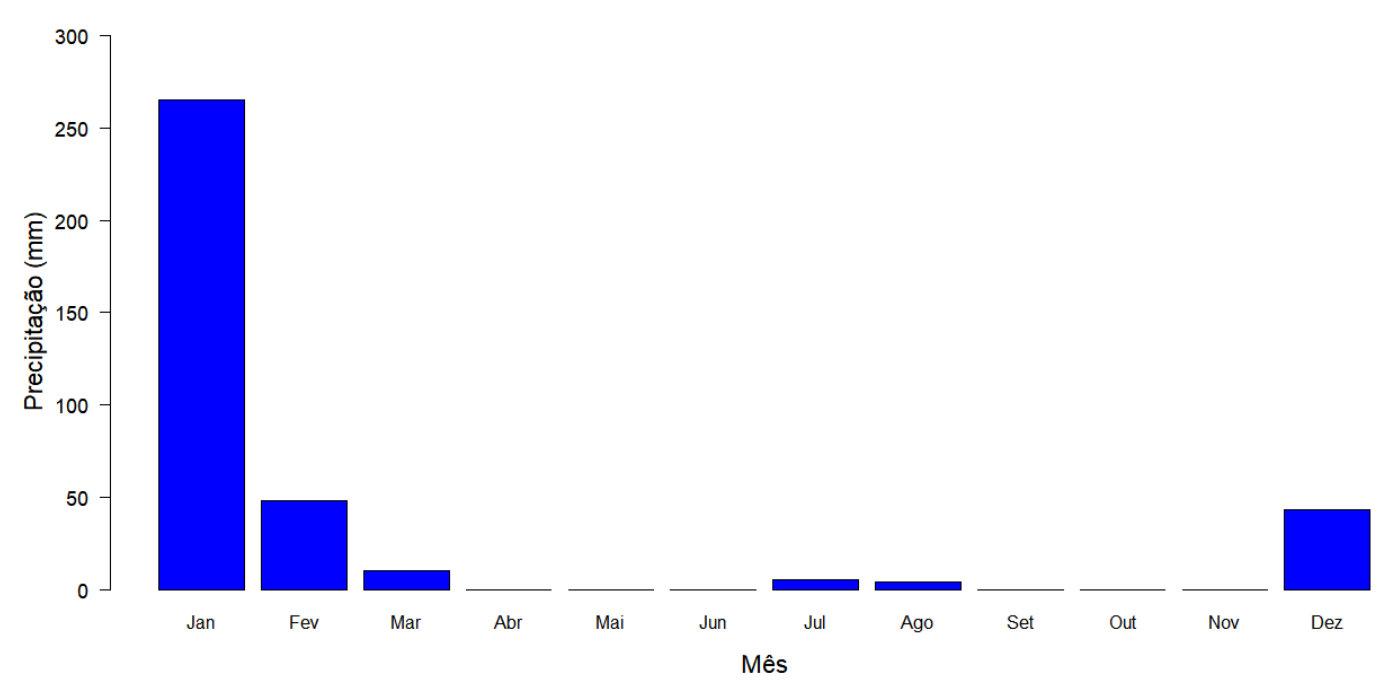

Figura 3. Estimativa de precipitação pluviométrica mensal estimada pelo SWAT para a bacia do pontal, 2016.

Variação da evapotranspiração obtida por meio do sensor MODIS

A variabilidade de estimativa da evapotranspiração ao longo dos meses durante o ano de 2016 está apresentada na Figura 4. Ressalva-se que houve dificuldades por conta da alta quantidade de nuvens sobre a imagem, que mesmo sendo feita uma estimativa mensal, não se conseguiu obter dados para todos os pixels, e assim, tendo valores muito baixos de evapotranspiração. Desta forma, não se obteve dados confiáveis para o mês de janeiro.

Os meses de fevereiro, março e abril foram os meses que se mostraram com maior quantidade de evapotranspiração, ten do valores médios de $59,47 \mathrm{~mm} ; 57,26 \mathrm{~mm}$ e $37,02 \mathrm{~mm}$, respectivamente. Esses valores podem estar relacionados aos volumes de chuvas que ocorreram, pois é considerado período chuvoso na região. Por outro lado, nos meses de maio, ju nho, julho, agosto, setembro e outubro foram observados baixos valores de evapotranspiração, sendo eles 25,09 mm; 18,15 mm; 13,22 mm; 9,39 $\mathrm{mm} ; 7,58 \mathrm{~mm}$ e $9,26 \mathrm{~mm}$, respectivamente. Esses meses são considerados de estiagem na região semiárida nordestina, o que pode justificar os baixos valores de evapotranspiração.

Mesmo os meses de novembro e dezembro tendo valores considerados baixos, pode-se observar um pequeno aumento na

Brito., P., V., Miranda., R., Q., Morais., Y., C., B., Costa., V., S., O., Moura., M., S., B., Silva., A., S., A., Galvincio., J., D. 
Revista Brasileira de Geografia Física v.14, n.05 (2021)2866-2877.

evapotranspiração em relação aos três últimos meses anteriores, tendo em média $11,59 \mathrm{~mm}$ e $10,75 \mathrm{~mm}$ respectivamente. Justamente, o período indicado como o início das chuvas na região.

Segundo Silva (2017), o índice de aridez no semiárido é superior a 1,0 para tod a sua área, desta forma a evapotranspiração na região é cerceada pela disponibilidade de chuva no local.

Algumas partes da região Sul da bacia, se mantém com um alto valor de evapotranspiração por todo o ano, isso ocorre por serem áreas de cultivos irrigados. Já em algumas regiões consideradas de Caatinga densa, a evapotranspiração alta tem maior duração de meses, e no período de chuvas cresce rapidamente. Ao Norte, podemos observar as menores quantidades, considerando que ocorrem por conta da maior área de agropecuária. E nas áreas de Caatinga densa degradada, que predomina nabacia, a evapotranspiração diminui rapidamente.

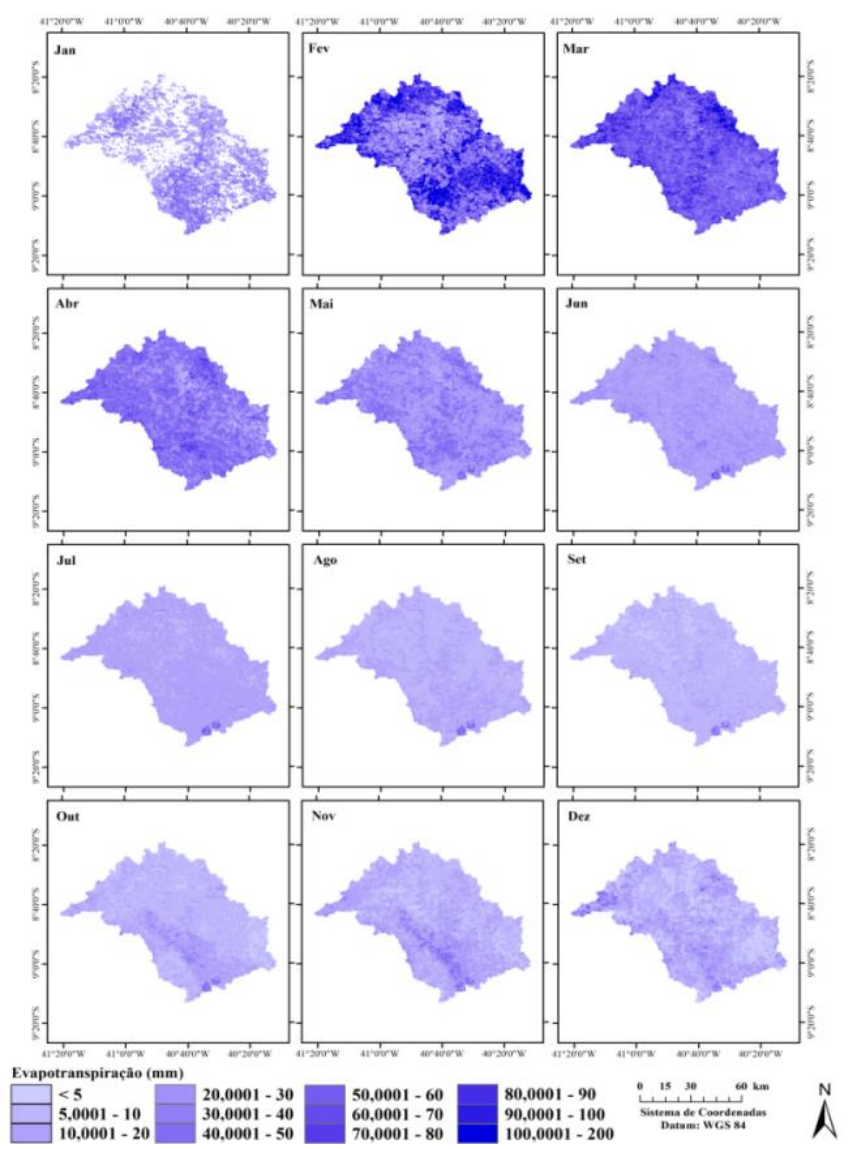

Figura 4. Variabilidade da evapotran spiração mensal para a bacia hidrográfica do riacho Pontal no ano de 2016.

Valores médios mensais de evapotranspiração do Modelo SWAT

Na Figura 5 estão representados os dados de evapotranspiração simulados do SWAT. A variação espaço-temporal da ETr na Bacia do Riacho Pontal foi de 2,76 a 42,39 mm, resultados semelhantes ao obtido com a utilização de produtos MODIS, estando os meses de janeiro, fevereiro, março e abril com maior evapotranspiração. No mês de dezembro, temos também um número alto de evapotranspiração obtido no SWAT, porém no MODIS os valores obtidos são considerados baixos. Em novembro quando se inicia o inverno e as chuvas começam a cair, pode-se observar que a evapotranspiração começa a crescer rapidamente, entretanto no produto do MODIS, o crescimento não ocorre de maneira tão acelerada. No mês de maio, que é o término do período de chuvas, a evapotranspiração começa a diminuir de forma rápida, como ocorreu também nos valores obtidos do MODIS.

As diferenças apresentadas nos resultados decorrentes do produto MODIS e a simulação do SWAT podem ocorrer pelo fato de que a radiação global medida em estações meteorológicas poder 
Revista Brasileira de Geografia Física v.14, n.05 (2021) 2866-2877.

está menor no decorrer de dias nublados, o que pode gerar interferência na quantidade de energia disponível no processo de evapotranspiração. Outros possíveis motivos na desigualdade dos dados, é que a evapotranspiração obtida pelo sensor MODIS utiliza o banco de dados Global Modeling and Assimilation Office (GMAO), com dados globais de clima considerados de resolução espacial baixa, além de incluir índices de vegetação no algoritmo de backup, que tende a aumentar ainda mais a incerteza. Desta forma, para estes tipos de medições do solo, mesmo com dias nublados se é produzido dados confiáveis, pois os dados de superfície são obtidos abaixo das nuvens, o que é contrário a dados ad quiridos em satélites, ou seja, os dados de superfície são obtidos a acima das nuvens, podendo não ter tanta precisão.

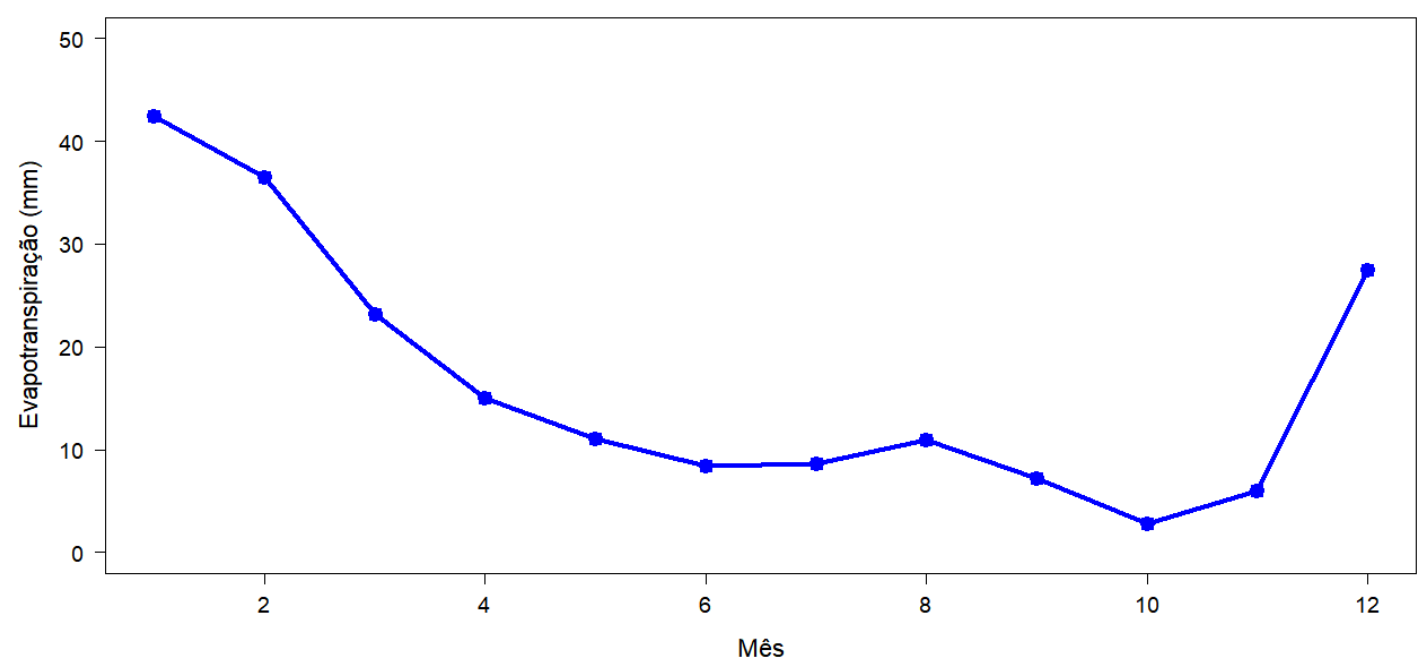

Figura 5. Estimativa da evapotranspiração mensal obtida por meio do Modelo SWAT para a bacia hidrográfica do riacho Pontal no ano de 2016.

Variação do escoamento superficial obtida por meio do modelo SWAT

A variabilidade de estimativa do escoamento superficial no decorrer dos meses durante o ano de 2016 está apresentada na Figura 6. Na qual, podemos observar que os meses de janeiro, fevereiro, março, agosto e dezembro foram os meses que apresentaram os maiores valores de escoamento superficial na área observada, tendo valores médios de $11,71 \mathrm{~mm}$; $2,96 \mathrm{~mm} ; 2.49 \mathrm{~mm} ; 3.38 \mathrm{~m}$ e $3.36 \mathrm{~mm}$, respectivamente. Sendo estes valores relacionados com o volume de precipitação na região. Já nos meses de abril, maio, junho, julho, setembro, outubro e novembro foram apontados valores baixos, tendo em média $0,97 \mathrm{~mm} ; 0,00 \mathrm{~mm} ; 0,00$ $\mathrm{mm} ; 0,00 \mathrm{~mm} ; 0,00 \mathrm{~mm} ; 0,00 \mathrm{~mm}$ e $0,09 \mathrm{~mm}$ na devida ordem. Sendo esse período tido como de estiagem no semiárido nordestino.

Na região Norte da bacia pode-se observar que ocorre um maior escoamento que nas áreas consideradas de agropecuária e solo exposto. Isto ocorre pelo fato de ter pouca vegetação, ou não ter vegetação na região. Desta forma, a água que cai sobre o solo pode escorrer facilmente. Podemos notar também que o sul da bacia dispõe de um valor considerado alto nas áreas de agricultura irrigada ou próximo delas, podendo ter como causa o solo já contido com a quantidade necessária de água vinda da irrigação, a pouca vegetação ou falta de vegetação.

Brito., P., V., Miranda., R., Q., Morais., Y., C., B., Costa., V., S., O., Moura., M., S., B., Silva., A., S., A., Galvincio., J., D. 


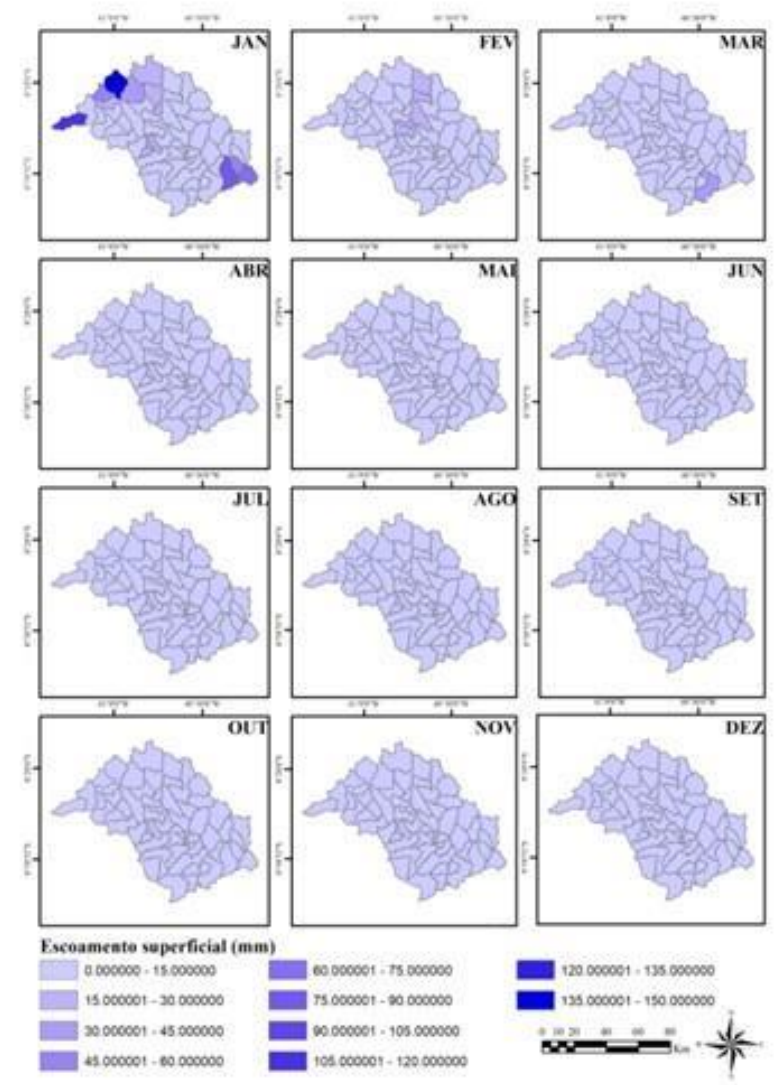

Figura 6. Variabilidade do escoamento superficial mensal para a bacia hidrográfica do riacho Pontal no a no de 2016.

Valores médios mensais de escoamento superficial

O escoamento superficial para o ano de 2016 variou de 0,00 a 40,20 mm (Figura 7), apresentando valores diferentes de zero apenas para janeiro, fevereiro, março e dezembro. Sen do estes apontados com números maiores de chuvas na região. Desta forma, temos também que os demais meses não dispõem de um número necessário de precipitação para ocorrer o escoamento.

Mesmo novembro e dezembro sendo o início do período de chuvas, o valor do escoamento é baixo, pois o solo se encontra muito seco infiltrando maior ou toda a quantidade de precipitação caída sobre ele. No entanto, em janeiro o solo se encontra saturado, ocorrendo maior escoamento sobre a superfície.
Percebe-se que o escoamento superficial tem sua dinâmica de crescimento e decrescimento semelhante com a da evapotranspiração, isto se justifica por ambas ser relacionadas com a precipitação ocorrida na região.

A relação do volume de chuva com a evapotranspiração e o escoamento superficial, também é mostrada no trabalho de Silva (2017). Segundo o autor, em torno de $23,4 \%$ do semiárido brasileiro a evapotranspiração demanda um valor acima de $95 \%$ do volume de chuva, que por consequência, tem um escoamento abaixo de $5 \%$ da chuva, que compromete a recarga dos reservatórios hídricos da região. $\mathrm{O}$ mes mo ainda conclui que os anos com volumes de chuvas superiores e inferiores à média, causam mudanças consideráveis tanto na evapotranspiração, qu an to no escoamento superficial.

Brito., P., V., Miranda., R., Q., Morais., Y., C., B., Costa., V., S., O., Moura., M., S., B., Silva., A., S., A., Galvincio., J., D. 


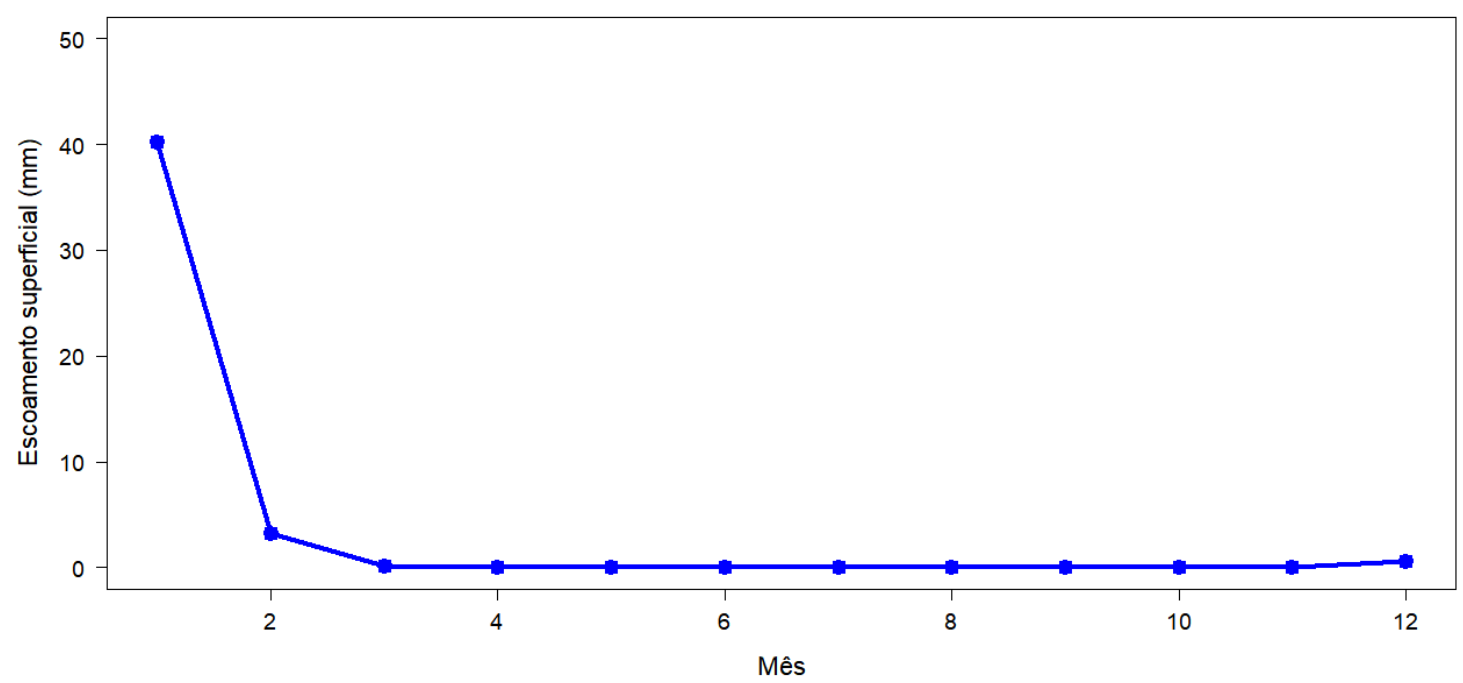

Figura 7. Escoamento superficial mensal estimado por meio do Modelo SWAT para a bacia hidrográfica do riacho Pontal no ano de 2016.

\section{Conclusões}

No ano de 2016 na bacia hidrográfica do riacho Pontal o mês que apresentou a maior evapotranspiração e escoamento superficial foi janeiro, tendo como principal causa o volume alto de precipitação. Já o mês que apresentou a menor evapotranspiração e escoamento superficial foi outubro, tendo como causa principal o volume baixo ou nulo de precipitação.

Foi possível constatar, que as maiores evapotranspiração ao longo do ano de 2016 ocorreram nas áreas de agricultura irrigada ten do como causa a irrigação constante, enquanto caatinga preservada foi devido a transpiração $d$ as plantas e armazenamento de água no solo. Já o escoamento superficial foi predominante nas áreas de agropecuária e solo exposto, tendo como causa principal a pouca ou falta de vegetação no local.

\section{Referências}

APAC. Agência Pernambucana de Águas e Clima, 2021. Bacias Hidrográficas - riacho do Pontal. Disponível: http://200.238.107.184/baciashidrograficas/40-bacias-hidrograficas/211bacia-do-riacho-pontal. Acesso: 16 jan. 2021. Arnold, J. G. et al., 2012. Swat: Model use, calibration, and validation. Transactions of

the ASABE, American Society of Agricultural and Biological Engineers, 55, 1491-1508.

Arnold, J.G., Srinivasan, R., Ramanarayanan, T.S., Williams, J., 1998. Large area hydrologic modeling and assessment: Part I - model development. Journal of American Water Resources Association 34, 73-90.

Bastiaanssen, W. G. M., 1995, Regionalization of surface flux densities and moisture in dicators in composite terrain. Ph.D. Thesis, Wageningen Agricultural University, Wageningen, Netherlands.

Brito, P.V.daS., Morais, Y.C.B., Ferreira, H.dosS., Silva, J.F., Galvíncio, J.D., 2017. Análise comparativa da umidade da vegetação de áreas de caatinga preservada, agricultura irrigada e sequeiro. Journal of Environ mental Analysis and Progress 3, 493-498.

Brutsaert, W.,2005. Hydrology: an in troduction. Cambridge University Press.

Bond, N.R., Burrows, R.M., Kennard, M.J., Bunn, S.E., 2019. Water scarcity as a driver of multiple stressor effects. In: Multiple stressors in river ecosystems. Elsevier. 111-129.

Vieira, P.deB., et al., 2016. Caatinga plants: Natural and semi-synthetic compounds potentially active against Trichomonas vaginalis. Bioorganic \& medicinal chemistry letters, 26, 2229-2236.

Cleugh, H.A., Leuning, R., Mu, Q., Running, S.W., 2007. Regional evaporation estimates from flux tower and MODIS satellite data. Remote Sensing of Environment 106, 285-304.

Fernandes, J. G., Galvíncio, J. D., \& dos Santos Freire, M. B. (2012). O Modelo SWAT e suas Possibilidades de Aplicações em APL Agrícolas. Revista Brasileira de Geografia Física, 5, 115-126.

Brito., P., V., Miranda., R., Q., Morais., Y., C., B., Costa., V., S., O., Moura., M., S., B., Silva., A., S., A., Galvincio., J., D. 
Ferreira, A.deN., Almeida, A.de, Koide, S., Minoti, R. T., Siqueira, M.B.B.de, 2021. Evaluation of Evapotranspiration in Brazilian Cerrado Biome Simulated with the SWAT Model. Water, 13(15), 2037.

Santos, L.A., Latrubesse, E.M., 2021. Aeolian mobility in the Middle São Francisco Dune Field, northeast Brazil, as a response to Caatinga's droughts and land-use changes. Geomorphology, p. 107940.

Saraiva, I., Fernandes, W., Naghettini, M., 2011. Simulação Hidrológica Mensal em Bacias Hidrográficas sem Monitoramento Fluviométrico. RBRH-Revista Brasileira de Recursos Hídricos, 16, 115-125.

Silva, E.R.A.C., Miranda, R.deQ., Ferreira, P.dosS., Gomes, V.P, Galvíncio, J.D., 2016. Estimativa do Estresse Hidrológico na Bacia Hidrográfica do Riacho do Pontal-PE. Caderno de Geografia, 26, 844-861.

Silva, J.R.I., Souza, R.M.S., Santos, W.A., Almeida, A.Q.de, Souza, E.S.de, Antonino, A.C.D., 2017. Aplicação do método de Budyko para modelagem do balanço hídrico no semiárido brasileiro. Scientia Plena, 13, n. 10.

Silva, J.M.C. da, Leal, I.R.; TABARELLI, M., 2018. Caatinga: the largest tropical dry forest region in South America. Springer.

Teixeira, A. H. D. C., Sherer-Warren, M., Hernandez, F. B., \& Lopes, H. L. (2012, October). Water productivity assessment by using MODIS images and agrometeorological data in the Petrolina municipality, Brazil. In Remote Sensing for Agriculture, Ecos ys tems, and Hydrology XIV (Vol. 8531, p. 85310G). International Society for Optics and Photonics.

Gash, J.H.C.,1979. An analytical model of rainfall interception by forests. Quarterly Journal of the Royal Meteorological Society, 105, 443, 43-55.

Galvincio, J. D. Luz, G. G. (2021). Desenvolvimento de Modelo que Estima o Impacto do $\mathrm{CO} 2$ Atmosférico nas Precipitações do Estado de Pernambuco, utilizando ARIMA. Revista Brasileira de Geografia Física, 14. 1840-1851. doi:https://doi.org/10.26848/rbgf.v14.4.p1840$\underline{1851}$

Galvíncio, J. (2021) Impacto do aumento de CO2 nas precipitações do estado de Pernambuco. Revista Brasileira de geografia Física, 14(3), 1828-1839.

Doi: https://doi.org/10.26848/rbgf.v14.3.p1828$\underline{1839}$
Galvíncio, Josiclêda Domiciano. Estimation of surface temperature with images obtained with drones, 2019. Joumal of Hyperspectral Remote Sensing, 9, 397-406.

Galvíncio, J.D.; Naue, C.R. Estimation of NDVI with visible images (RGB) obtained with drones, 2019. Journal of Hyperspectral Remote Sensing, 9, 407-420.

Gutiérrez, A.P.A., Engle, N.L., Nys, E.D., Molejón, C., Martins, E.S., 2014. Drought preparedness in Brazil. Weather and Climate Extremes, 3, 95-106.

Lenhart, T., Eckhardt, K., Fohrer, N., Frede, G., 2002. Comparison of two different approaches of sensitivity analysis. Physics and Che mistry of the Earth 27, 645-654

Lima, C.E.S.D., Costa, V.S.O., Galvíncio, J.D., Silva, R.M.D., Santos, C.A.G., 2021. Assessment of automated evapotranspiration estimates obtained using the GP-SEBAL algorithm for dry forest vegetation (Caatinga) and agricultural areas in the Brazilian semiarid region. Agricultural Water Management 250, 106863.

Marengo, J.A., 2010. Vulnerabilidade, impactos e adaptação à mudança do clima no semi-árido do Brasil. Parcerias estratégicas, 13, 149-176.

Melesse, A. M., Weng, Q., Thenkabail, P. S., \& Senay, G. B. (2007). Remote sensing sensors and applications in environmental resources mapping and modelling. Sensors, 7(12), 32093241.

Miranda, R.Q., Galvincio, J.D., Morais, Y.C.B., Moura, M.S.B., Jones, A., Srinivasan, R., 2018. Dry forest deforestation dynamics in Brazil's Pontal basin. Revista Caatinga 31, 385-395.

Miranda, R.Q. Avaliação integrada da variação espacial e temporal do balanço hídrico na Caatinga. Tese (Doutorado em Desenvolvimento e Meio Ambiente). Recife, UFPE.

MMA. Ministério do Meio Ambiente, 2010. Monitoramento dos Biomas Brasileiros: bioma Caatinga. MMA, Brasília.

Morais, Y.C.B, Araújo, M.doS.B.de, Moura, M.S.B.de, Galvíncio, J.D., Miranda, R.Q., 2017. Análise do Sequestro de Carbono em áreas de Caatinga do Semiárido Pernambucano. Revista Brasileira de Meteorologia, 32, 585-599, 2017. doi: https://doi.org/10.1590/0102-7786324007

$\mathrm{Mu}$, Q., Heinsch, F.A., Zhao, M., Running, S.W., 2007. Development of a global

Brito., P., V., Miranda., R., Q., Morais., Y., C., B., Costa., V., S., O., Moura., M., S., B., Silva., A., S., A., Galvincio., J., D. 
Revista Brasileira de Geografia Física v.14,n.05 (2021) 2866-2877.

evapotranspiration algorithm based on MODIS and global meteorology data. Remote Sensing of Environment 111, 519-536.

Novo, E. M. L. M., 2010. Sensoriamento Remoto: princípios e aplicações. $2^{\text {a }}$ edição. São Paulo: Edgard Blücher, v. 308.

Rallison, R.E., Miller, N., 1981. Past, present and future SCS runoff procedure, in: Singh, V.P.

Uzeika, Talita et al., 2012. Use of the SWAT model for hydro-sedimentologic simulation in a small rural watershed. Revista Brasileira de Ciência do Solo, 36, .557-565.

Elhakeem, M., Papaniclaou, A.N. Estimation of the Runoff Curve Number via Direct Rainfall Simulator Measurements in the State of Iowa, USA. Water Resour Manage 23, 2455-2473 (2009) https://doi.org/10.1007/s11269-0089390-1

Silva, B.B.da, Galvincio, J.D., Montenegro, S.M.G.L., Machado, C.C.C., Oliveira, L.M.M.de, Moura, M.S.B.de, 2013. Determinação por sensoriamento remoto da produtividade primária bruta do perímetro irrigado São Gonçalo-PB. Revista Brasileira de Meteorologia 28, 57-64. doi: $\quad$ https://doi.org/10.1590/S0102$\underline{77862013000100006}$
Souza, V.A.S., 2015. Monitoramento Hidrossedimentológico com Suporte do Modelo SWAT na Amazônia Ocidental Bacia do Rio Machadinho/RO. Programa de Pós-Graduação em Engenharia Civil. COPPE, UFRJ, Rio de janeiro.

Tyagi, R., Kathpalia, J., Chander, S., 2020. Comparative analysis between direct seeded rice and conventional transplanted rice method. The Pharma Innovation Joumal, 9(6), 236-238.

Wang, J., Chermak, J.M., 2021. Is less always more? Conservation, efficiency and water education programs. Ecological Economics, v. 184, p. 106994.

Brito., P., V., Miranda., R., Q., Morais., Y., C., B., Costa., V., S., O., Moura., M., S., B., Silva., A., S., A., Galvincio., J., D. 